\title{
Dental age estimation using incremental lines of cementum
}

\author{
Nupoor Kulkarni ${ }^{1}$, Swetha Mahesh ${ }^{2}$, Gokul Sridharan ${ }^{3, *}$ \\ ${ }^{1,2}$ Dental Surgeon, ${ }^{3}$ Associate Professor, Dept. of Oral Pathology and Microbiology, YMT Dental College and Hospital, Mumbai, \\ Maharashtra, India \\ *Corresponding Author: Gokul Sridharan \\ Email: drgokuls@gmail.com
}

\begin{abstract}
Forensic odontology is a specialty of dentistry which in the interest of justice deals with the examination of dental evidence for evaluation and presentation of the dental findings. An important aspect of forensic odontology is the identification of age, sex and race of an individual based on their dental characteristics. Various methods utilizing the dentin translucency, cemental annulations, attrition, pulp-tooth ratio, amino acid racemization among others are available for dental age estimation. The aim of the present study was to evaluate the efficiency of incremental lines of cementum in age estimation. Ten freshly extracted teeth were histologically observed using light microscope and image analysis software for assessment of incremental lines of cementum. The incremental lines were counted, and the age was estimated using a prescribed formula. The estimated age was then compared with the actual age of the individual to determine its efficiency. The preliminary study results indicated a positive correlation between estimated age and the actual age in $70 \%$ of the samples. Thus, it can be concluded that cemental annulations could serve as an important factor in age determination. Further analysis with larger sample size and advanced techniques will help in improving the accuracy of this method.
\end{abstract}

Keywords: Dental age estimation, Incremental lines of cementum, Forensic odontology.

\section{Introduction}

The specialty of forensic odontology has undergone significant advancements in the recent years. One of the reasons for this is the ability to utilize the dental hard and soft tissues for identification of race, sex and age in cases of mass disasters, identification of assault victims as well as for medico-legal issues. ${ }^{1}$ Identification of age is an important component of forensic odontology. ${ }^{2}$ Ever since the first description of utilizing the dental hard tissues for age estimation by Gustaffson, various methods have been researched and clinically evaluated. ${ }^{3}$ The use of dental hard tissues has found favor with forensic odontologists owing to its ability to resist degradation long after the other body tissues are lost. ${ }^{4}$ One of the methods of age estimation is by utilizing the cemental annulations or incremental lines of cementum. ${ }^{5}$ Cementum is a mineralized hard tissue of the tooth which undergoes incremental deposition at regular intervals and shows an increase in thickness between the age of 20 and $60 \mathrm{yrs} .{ }^{6}$ The increments of cementum deposition are delineated by incremental lines known as incremental lines of salter which can be used as a reliable tool for age estimation. ${ }^{7}$ Unlike other dental hard tissues, cementum has a unique position in the alveolar process and thus is less exposed to the outer environmental surroundings, thereby increasing its accuracy towards estimating age of an individual. ${ }^{8}$ Thus, the aim of the study was to perform a qualitative analysis of incremental lines of cementum to determine their effectiveness in dental age estimation.

\section{Materials and Methods}

The study sample included extracted tooth specimen which were extracted advised for removal secondary to orthodontic treatment or those affected by dental caries $(n=10)$. The samples included in the study was in the age range of 15 to 61 years of age. Single rooted teeth were preferred for ease of assessing the incremental lines of cementum. Longitudinal ground sections of the tooth specimen were made and observed under light microscope. Following preparation of the sections, photomicrographs were taken and the number and distance between incremental lines of cementum were analyzed using image analysis software (Motic software). Following this, the number of incremental line of Salter (N) was calculated using the formula $\mathrm{N}=\mathrm{X} / \mathrm{Y}$.

Where, $\mathrm{N}$ is the number of incremental lines of cementum; $\mathrm{X}$ is the total width of cementum from cemento-dentinal junction to surface and $\mathrm{Y}$ is the distance between adjacent lines of Salter. The incremental lines were mainly analyzed in the cervical and middle third of the root.

Further, the dental age was estimated using the formula $\mathrm{E}=\mathrm{N}+$ eruption age of the tooth (6), where $\mathrm{E}$ is the dental age and $\mathrm{N}$ is the number of incremental lines of cementum. For calculation of dental age, the investigators were blinded with respect to the actual age of the tooth to prevent any observer bias. The estimated age as per the formula was further compared with the actual age to determine the accuracy of the age determination.

\section{Results}

The obtained age based on the formula and the actual age was compared and the difference in the age determination was noted (Table 1). From the tooth specimen analyzed, it was noted that that $70 \%$ of the sample had a positive correlation between the estimated age and the chronological age. And $30 \%$ of the sample had a negative correlation. The correlation was found out 
on the basis of mean age difference. The wide difference between the actual and estimated age in some of the samples could be due to the older age group of the tooth specimen.

Table 1: Age Estimation using incremental lines of salter and its comparison with the actual chronological age

\begin{tabular}{|l|c|c|c|c|c|c|c|}
\hline Sample No. & Tooth no. & $\mathbf{X}$ & $\mathbf{Y}$ & $\mathbf{N}=\mathbf{X} / \mathbf{Y}$ & Actual age & Estimated age & Difference \\
\hline 1 & 22 & 104.1 & 4 & 26.02 & 61 years & 34 years & 27 years \\
\hline 2 & 23 & 340 & 23.3 & 14.6 & 61 years & 26.5 years & 34.5 years \\
\hline 3 & 24 & 142 & 8 & 17.75 & 61 years & 29.75 years & 31.25 years \\
\hline 4 & 11 & 43.3 & 7.2 & 6.01 & 19 years & 23 years & 4 years \\
\hline 5 & 44 & 46.8 & 10.8 & 4.3 & 15 years & 15.3 years & 0.3 years \\
\hline 6 & 25 & 174.4 & 12.2 & 14.3 & 16 years & 25.2 years & 9.2 years \\
\hline 7 & 45 & 79.6 & 4.5 & 17.7 & 15 years & 28 years & 13 years \\
\hline 8 & 21 & 152 & 8 & 19 & 28 years & 26 years & 2 years \\
\hline 9 & 35 & 86.1 & 2.1 & 41 & 56 years & 51 years & 5 years \\
\hline 10 & 13 & 168 & 4.2 & 40 & 56 years & 52 years & 4 years \\
\hline
\end{tabular}

\section{Discussion}

Dental age estimation is an important part of forensic odontology. The different methods of age estimation employed various characteristics of the tooth such as dentin translucency, attrition of enamel, pulproot ratio, cemental annulations and amino acid racemization among others. The present study was a preliminary study to assess the utility of incremental lines of cementum in dental age estimation. The basis of utilizing cemental annulations for dental age estimation was that cementum appears as a series of alternating light and dark bands and each one light-dark band was considered as one annulation which corresponds to oneyear age of the individual. Adding these lines in thin histological sections and adding them to mean eruption age of the tooth will give the chronological age of the individual. $^{2}$

In the present study dental age estimation using incremental lines of cementum was done in 10 freshly extracted single rooted teeth that were grounded and analyzed using light microscope and motic image analysis software. The age range of the analyzed samples was between 15 to 61 years. Most of the samples $(n=7)$ showed a close correlation of the estimated age to the actual age. The area of assessing the incremental lines of cementum was mainly at the middle and the cervical third of the cementum because presence of acellular cementum makes it easier and clearer to count the incremental lines of Salter; apical third contains cellular cementum and may be mistaken in cases of hypercementosis or root resorption or periapical pathologies. ${ }^{9}$

Bhondey et al (2015) evaluated the dental age using incremental lines of cementum and concluded that the counting incremental lines in the middle third of the root and the use of phase contrast microscopy improved the accuracy of age estimation and showed a significant correlation between the predicted age and the actual chronological age of the individual. ${ }^{10}$ Meinl et al (2008) evaluated the dental age estimation using different methods and concluded that age estimation using tooth cemental annulations was more predictable although use of a standardized procedure would help in enhancing its efficiency. ${ }^{11}$ Aggrawal et al (2008) showed a significant correlation between the predicted age and the chronological age of the individual thereby suggesting that cemental annulations is a moderately reliable indicator of age estimation. ${ }^{8}$ Other literature data also suggested the clinical utility of cemental lines in dental age estimation albeit with varying success. ${ }^{6,12,13}$

The use of cemental annulations for dental age estimation has been successfully analyzed and reported in the literature. Nevertheless, there are few drawbacks associated with its use. Cemental annulations are affected by periodontal diseases, systemic conditions and age which may negate their use as accurate predictor of dental age. Periodontal inflammation and subsequent disease may result in decrease in the functional significance of Sharpeys fibers due to reducing alveolar bone. As cemental annulations are interrelated with sharpeys fibers, an arrest of cemental annulation process may occur thereby affecting the age estimation. ${ }^{8}$ The predictability of age was found to be effective in younger age groups than in individuals with over 60 years of age. The lower correlation in older persons could be due to decreased apposition of cemntum over 60 years of age. ${ }^{14}$ Also, cementum deposition is altered in systemic conditions such as diabetes and hypophosphotasia which could influence the accuracy of age estimation.

\section{Conclusion}

To conclude, this preliminary study of using cemental annulations indicate that they can be considered as one of the important methods for dental age estimation in forensic odontology. While the study results were promising, it had certain drawbacks such as the insufficient sample size and the use of conventional armamentarium. Use of specialized microscopy such as polarizing microscope or electron microscope in a larger sample size is necessary for validating the study findings. Further, it is necessary to standardize the evaluation and analytical procedure for better sensitivity and specificity in clinical applications. 


\section{References}

1. Alghonamy WY, Gaballah OM, Labah DA. Age estimation in adult human sound and periodontally affected teeth using tooth cementum annulations. Tanta Dental Journal. 2015;12:277-85.

2. Dias PEM, Beaini TL, Melani RFH. Age estimation from dental cementum incremental lines and periodontal disease. J Forensic Odontostomatol. 2010;28:13-21

3. Gustaffson G. Age determination on teeth. $J$ Am Dent Assoc. 1950;41:45-54

4. Stein TJ. Anatomy of root apex and histological changes with age. Oral surgery oral medicine oral pathology. 1990;69:266-70

5. Stott GG, Sis RF, Levy BM. Cementum annulations as an age criterion in forensic dentistry. J Dent Res. 1982;61:814-7

6. Pundir S, Saxena S, Aggrawal P. Estimation of age based on tooth cementum annulations using three different microscopic methods. Journal of forensic dental sciences. 20019;1:82-7

7. Swetha G, Kattappagari KK, Poosarla CS, Chandra LP, Gontu SR, Badam VR. Quantitative analysis of dental age estimation by incremental line of cementum. $J$ Oral Maxillofac Pathol. 2018;22:138-42.

8. Aggrawal P, Saxena S, Bansal P. Incremental lines in root cementum of human teeth: An approach to their role in age estimation using polarizing microscopy. Indian $J$ Dent Res. 2008;19:326-30.

9. Cipriano A. Cold stress in captive great apes recorded in incremental lines of dental cementum. Folia Prim atol (basel). 2001;73:21-31.

10. Bhondey A, Thakur M, Palve D, Dhengar YS, Bhagwatkar T, Chaturvedi S. Age revealing annulations: application for estimation of age in central India population. Annals of Dental Specialty. 2015;3:96-9.

11. Meinla A, Huber CD, Tangl S, Gruber GM, TescherNicola M, Watzek G. Comparison of validity of three dental methods for the estimation of age at death. Forensic Sci Int. 2008;178:96-105.

12. Gupta P, Kaur S, Madhu Shankari GS, Jawanda MK, Sahi N. Human age estimation from tooth cementum and dentin. J Clinc Diagn Res. 2014;8:ZC07- ZC10.

13. Padavala $S$, Gheena $S$. Estimation of age using cemental annulations. J Pharm Sci \& Res. 2015;7:461-463.

14. Solheim T. Dental cementum apposition as an indicator of age. Scand J Dent Res. 1990;98:510-9. 